\title{
POLYNOMIAL COMPUTATION OF HANKEL SINGULAR VALUES
}

\author{
Huibert Kwakernaak \\ Systems and Control Group, Department of Applied Mathematics \\ University of Twente, P. O. Box 217, 7500 AE Enschede, The Netherlands \\ Fax Intl+31-53-340733, E-mail h.kwakernaakemath.utwente.nl
}

\begin{abstract}
The paper presents a revised and improved version of a polynomial algorithm published by N. J. Young in $\mathbf{1 9 9 0}$ for the computation of the singular values and vectors of the Hankel operator defined by a linear time-invariant system with rational transfer matrix.
\end{abstract}

\section{Introduction}

We describe an algorithm for the computation of the Hankel singular values and vectors by polynomial methods. It is a much streamlined version of that of Young [1]. The computation of Hankel singular values arises in determining lower-order approximations of linear multivariable systems [2], and in $\mathcal{H}_{\infty}$ optimization [3]. We consider continuous- time systems rather than discrete-time systems as Young [1] does. The results can easily be adapted to the discretetime case.

Let $G$ be the $n \times m$ strictly proper transfer matrix of a strictly stable continuous-time system, with left and right coprime polynomial matrix fraction representations

$$
G=D^{-1} N=\bar{N} \bar{D}^{-1}
$$

with $D$ row reduced and $\bar{D}$ column reduced. The Hankel operator $G_{\mathrm{H}}$ defined by $G$ maps any finite-energy input $u$ such that $u(t)=0$ for $t \geq 0$ to the finite-energy signal $y_{+}$that is obtained from the corresponding output $y$ as

$$
y_{+}(t)= \begin{cases}0 & \text { for } t<0 \\ y(t) & \text { for } t \geq 0\end{cases}
$$

The Hankel singular values of $G$ are the singular values of the Hankel operator $G_{H}$.

Good references for the polynomial methods used in this paper are the books by Kučera [4], Kailath [5], and Callier and Desoer [6].

\section{Representation of the Hankel Operator in Expedient Bases}

Following Young [1], we successively consider the kernel (or nullspace,) the cokernel and the range of the Hankel operator $G_{H}$, and find suitable bases for the latter two spaces.

The kernel of the Hankel operator consists of all inputs in the domain of $G_{\mathrm{H}}$ that are mapped to the zero signal. The cokernel of $G_{H}$ consists of all signals $u$ in the domain of $G_{\mathrm{H}}$ that are $o r$ thogonal to the kernel. It is not difficult to argue that the cokernel of $G_{\mathrm{H}}$ is a finite-dimensional signal space, whose basis may conveniently be chosen as the signals whose (two-sided) Laplace transform is given by

$$
\bar{D}^{\sim}(s)^{-1} e_{i} s^{k},
$$

$k=0,1, \cdots, c_{i}-1, i=1,2, \cdots, m$, where $c_{i}$ is the degree of the $i$ th column of $\bar{D}$, and $e_{i}$ the $i$ th $m$-dimensional unit vector. The tilde denotes the adjoint. Juxtapose these basis vectors to form the rational matrix

$$
\begin{array}{r}
\bar{D}^{\sim}(s)^{-1}\left[\begin{array}{lllll}
e_{1} & e_{1} s & \cdots & e_{1} s^{c_{1}-1} & e_{2} \\
e_{3} & \cdots & e_{m} s^{c_{m}-1}
\end{array}\right]=\bar{D}^{\sim}(s)^{c_{2}-1} V(s),
\end{array}
$$

with $V$ a polynomial matrix. Then our basis for the cokernel of $G_{H}$ consists of those signals whose Laplace transforms are the columns of 
$\mathcal{D}=\left(\bar{D}^{\sim}\right)^{-1} V$. Young [1] calls this an expedient basis. The Laplace transform of any element $u$ in the cokernel of $G_{\mathrm{H}}$ may be represented as $\hat{u}=\mathcal{D} v$, with $v$ a suitable constant finite-dimensional vector.

Given any such input $u$ in the cokernel of $G_{H}$, the corresponding output $y$ has Laplace transform

$$
\hat{y}=G \hat{\imath}=D^{-1} N\left(\bar{D}^{\sim}\right)^{-1} V v
$$

We determine the corresponding truncated output $y_{+}$by partial fraction expansion. Define the right coprime polynomial matrices $\tilde{V}$ and $\tilde{D}$ by the left-to-right conversion

$$
\left(\bar{D}^{\sim}\right)^{-1} V=\tilde{V} \tilde{D}^{-1}
$$

and perform the partial fraction expansion

$$
D^{-1} N \tilde{V} \tilde{D}^{-1}=D^{-1} A+B \tilde{D}^{-1} \text {. }
$$

The polynomial coefficient matrices $A$ and $B$ may be obtained by solving the bilateral linear polynomial matrix equation

$$
N \tilde{V}=A \tilde{D}+D B
$$

It follows that

$$
\hat{y}=D^{-1} A v+B \tilde{D}^{-1} v
$$

Since $D^{-1}$ has all its poles in the left-half plane and $\tilde{D}^{-1}$ has all its poles in the right-half plane, we immediately conclude that the Laplace transform $\hat{y}_{+}$of $y_{+}$is

$$
\hat{y}_{+}=D^{-1} A v
$$

Hence, a convenient basis for the range of the Hankel operator consists of the signals whose Laplace transforms are the columns of the rational matrix $\mathcal{R}=D^{-1} A$. Moreover, in terms of the bases we selected for the cokernel and range, the Hankel operator simply is represented by the unit matrix.

Our approach differs from that of Young [1] in the choice of the basis for the range of the Hankel operator.

\section{Computation of the Hankel Singular Values and Vectors}

Let $\Gamma_{d}$ be the Gramian of the basis selected for the cokernel (consisting of the columns of $\mathcal{D}$ ), and $\Gamma_{r}$ the Gramian of the basis for the range (consisting of the columns of $\mathcal{R}$.) The computation of these Gramians is detailed later. Again following Young [1], we factor the Gramians as

$$
\Gamma_{d}=T_{d}^{\mathrm{T}} T_{d}, \quad \Gamma_{r}=T_{r}^{\mathrm{T}} T_{r}
$$

Then the singular values of the Hankel operator are the singular values of the matrix $T_{r} T_{d}^{-1}$.

Summary (Computation of Hankel singular values.) The singular values of the Hankel operator $G_{\mathrm{H}}$ defined by the system with the strictly stable and strictly proper $n \times m$ transfer matrix $G=D^{-1} N=\bar{N} \bar{D}^{-1}$, with $D$ row reduced and $\bar{D}$ column reduced, may be found as follows.

1. Form the polynomial matrix $V$ by juxtaposing the columns $e_{i} s^{k}, k=0,1, \cdots, c_{i}-1$, $i=1,2, \cdots, m$, with $e_{i}$ the $i$ th $m$ dimensional unit vector and $c_{i}$ the degree of the $i$ th column of $\bar{D}$. Compute the right coprime polynomial matrices $\tilde{V}$ and $\tilde{D}$, with $\tilde{D}$ column reduced, by the left-to-right conversion

$$
\left(\bar{D}^{\sim}\right)^{-1} V=\tilde{V} \tilde{D}^{-1}
$$

2. Solve the bilateral linear polynomial equation

$$
N \tilde{V}=A \tilde{D}+D B
$$

for polynomial matrices $A$ and $B$ such that $D^{-1} A$ is strictly proper.

3. Compute the Gramian $\Gamma_{d}$ of the signals whose Laplace transforms are the columns of $\mathcal{D}=\tilde{V} \tilde{D}^{-1}$. Also compute the Gramian $\Gamma_{r}$ of the signals whose Laplace transforms are the columns of $\mathcal{R}=D^{-1} A$.

4. Factor the Gramians as

$$
\Gamma_{d l}=T_{d}^{\mathrm{T}} T_{d}, \quad \Gamma_{r}=T_{r}^{\mathrm{T}} T_{r}
$$


and compute the singular value decomposition

$$
T_{r} T_{d}^{-1}=U \Sigma W^{\mathrm{H}} .
$$

5. The singular values of the Hankel operator are the diagonal entries of $\Sigma$. Its right and left singular vectors are the columns of

$$
\mathcal{D} T_{d}^{-1} W \text { and } \quad \mathcal{R} T_{c}^{-1} U
$$

respectively.

\section{Computation of the Gramians}

It remains to calculate the Gramians. Here we again offer an alternative to Young's proposal [1]. Consider computing the Gramian $\Gamma$ of a finite number of time signals $x_{i}, i=1,2, \cdots, p$, whose Laplace transforms $\hat{x}_{i}, i=1,2, \cdots, p$, are the columns of the strictly stable and strictly proper rational matrix

$$
X=Q R^{-1},
$$

with $Q$ and $R$ right coprime such that $R$ is column reduced. The $(i, k)$ element $\Gamma_{i k}$ of $\Gamma$ is the inner product

$$
\begin{aligned}
\Gamma_{i k} & =\int_{-\infty}^{\infty} x_{i}^{\mathrm{H}}(t) x_{k}(t) d t \\
& =\int_{-\infty}^{\infty} \hat{x}_{i}^{\mathrm{H}}(j 2 \pi f) \hat{x}_{k}(j 2 \pi f) d f .
\end{aligned}
$$

It follows that

$$
\begin{aligned}
\Gamma & =\int_{-\infty}^{\infty} X^{\sim}(j 2 \pi f) X(j 2 \pi f) d f \\
& =\int_{-\infty}^{\infty}\left(R^{\sim}\right)^{-1} Q^{\sim} Q R^{-1} d f
\end{aligned}
$$

where for simplicity we suppress the argument $j 2 \pi \int$. Perform the partial fraction expansion

$$
\left(R^{\sim}\right)^{-1} Q^{\sim} Q R^{-1}=\left(R^{\sim}\right)^{-1} C^{\sim}+C R^{-1},
$$

where the polynomial coefficient matrix $C$ is a solution of the symmetric bilateral polynomial matrix equation

$$
Q^{\sim} Q=R^{\sim} C+C^{\sim} R
$$

such that $C R^{-1}$ is strictly proper. It follows that

$$
\Gamma=\int_{-\infty}^{\infty}\left[\left(R^{\sim}\right)^{-1} C^{\sim}+C R^{-1}\right] d f .
$$

Since by assumption $R$ is strictly Hurwitz, $C R^{-1}$ is the (two-sided) Laplace transform of a matrix function $\gamma$ such that $\gamma(t)=0$ for $t<0$, and

$$
\Gamma=\lim _{t \downarrow 0} \gamma(t)
$$

By the initial value theorem,

$$
\lim _{t ! 0} \gamma(t)=\lim _{|s| \rightarrow \infty} s C(s) R^{-1}(s) .
$$

Define $R_{l}$ as the leading coefficient matrix of the column reduced matrix $R$, and $C_{l}$ as the associated leading coefficient matrix ${ }^{1}$ of $C$. Then,

$$
\Gamma=C_{l} R_{l}^{-1} \text {. }
$$

If $R$ is strictly anti-Hurwitz the Gramian of the functions whose Laplace transforms are the columns of $Q R^{-1}$ may be found as the Gramian of the functions whose Laplace transforms are the columns of $X$ defined by $X(s)=$ $Q(-s) R^{-1}(-s)$.

\section{Summary (Computation of the Gramian.)} Consider the signals whose Laplace transforms are the columns of the strictly stable and strictly proper rational matrix $Q R^{-1}$ with the polynomial matrix $Q$ and $R$ right coprime such that $R$ is column reduced. The Gramian $\Gamma$ of these signals may be computed as follows.

1. Solve the symmetric bilateral linear polynomial matrix equation

$$
Q^{\sim} Q=R^{\sim} C+C^{\sim} R
$$

for the square polynomial matrix $C$ such that $C R^{-1}$ is strictly proper.

2. Let $R_{l}$ be the leading coefficient matrix of the column reduced polynomial matrix $R$, and $C_{l}$ the associated leading coefficient matrix of $C$. Then the Gramian $\Gamma$ is

$$
\Gamma=C_{l} R_{l}^{-1}
$$

\footnotetext{
${ }^{1}$ Let the column degrees of $R$ be given by $c_{1}, c_{2}, \cdots, c_{p}$. Then the $k$ th column of $C_{l}$ is the $k$ th column of the coefficient matrix of $C$ corresponding to $s^{c_{k}-1}$.
} 


\section{Implementation}

Implementation of the algorithm under MAT$L A B$ first of all requires a way of representing polynomial matrices, and basic routines for elementary polynomial matrix manipulations such as addition and multiplication. More advanced routines are needed for left-to-right and right-to-left conversion of polynomial matrix fractions, transformation to row- and columnreduced form, and the solution of symmetric and asymmetric bilateral linear polynomial matrix equations. Several of these routines were already available [7] and others have been developed (in particular, for the solution of bilateral equations.)

Left-to-right and right-to-left conversion may be done by Kailath's algorithms ([5], Section 6.7.) Callier [8] describes how to transform to row- and column-reduced form. A provisional and rather inefficient way of solving bilateral equations is to expand them in terms of linear coefficient equations.

\section{Example}

We consider Example 6.1 of Glover [2], where

$$
\begin{aligned}
& G(s)=\left[\begin{array}{cc}
\frac{.45+2 s}{.09+1.25 s+s^{2}} & 0 \\
0 & 1 \\
.5+s
\end{array}\right] \\
& =\underbrace{\left[\begin{array}{cc}
.09+1.25 s+s^{2} & 0 \\
0 & .5+s
\end{array}\right]^{-1}}_{D(s)} \underbrace{\left[\begin{array}{cc}
.45+2 s & 0 \\
0 & 1
\end{array}\right]}_{N(s)} \\
& =\underbrace{\left[\begin{array}{cc}
0 & .45+2 s \\
1 & 0
\end{array}\right]}_{N(s)} \underbrace{\left[\begin{array}{cc}
0 & .09+1.25 s+s^{2} \\
.5+s & 0
\end{array}\right]^{-1}}_{\bar{D}(s)} .
\end{aligned}
$$

Since the column degrees of $\bar{D}$ are $c_{1}=1$ and $c_{2}=2$ we have

$$
V(s)=\left[\begin{array}{lll}
1 & 0 & 0 \\
0 & 1 & s
\end{array}\right] .
$$

By the left-to-right conversion (12) we obtain

$$
\tilde{V}(s)=\left[\begin{array}{rrr}
0 & 0 & 1 \\
-1 & 0 & 0
\end{array}\right],
$$

$$
\tilde{D}(s)=\left[\begin{array}{ccc}
-.5+s & 0 & 0 \\
0 & s & .09 \\
0 & -1 & -1.25+s
\end{array}\right]
$$

Solution of the bilateral polynomial matrix equation (13) results in

$$
\begin{aligned}
& A(s)=\left[\begin{array}{ccc}
0 & 1.7+2 s & -.18-.8 s \\
1 & 0 & 0
\end{array}\right], \\
& B(s)=\left[\begin{array}{rrr}
0 & -2 & .8 \\
-1 & 0 & 0
\end{array}\right] .
\end{aligned}
$$

The two Gramians may be found to be

$$
\begin{aligned}
& \Gamma_{c}=\left[\begin{array}{ccc}
1 & 0 & 0 \\
0 & 4.44444 & 0 \\
0 & 0 & 0.4
\end{array}\right], \\
& \Gamma_{r}=\left[\begin{array}{ccc}
1 & 0 & 0 \\
0 & 14.4444 & -2 \\
0 & -2 & 0.4
\end{array}\right],
\end{aligned}
$$

resulting in

$$
T_{r} T_{c}^{-1}=\left[\begin{array}{ccc}
1 & 0 & 0 \\
0 & 1.8028 & -.8321 \\
0 & 0 & .5547
\end{array}\right] .
$$

The singular values of this matrix are the correct numbers 2,1 , and 0.5 .

\section{Concluding Remarks}

The revised algorithm for the polynomial computation of the singular values of the Hankel operator is considerably more polished than Young's original algorithm [1].

Whether it can compete with state space algorithms remains to be seen. Tentative numerical experiments indicate that for high-order systems scaling of the polynomial matrices $N$ and $D$ (so that the constant and leading coefficient matrices are of the same order of magnitude) is mandatory, and that solution of bilateral linear polynomial matrix equations by coefficient expansion is highly inefficient. 


\section{References}

[1] N. J. Young, "A polynomial method for the singular value decomposition of block Hankel operators," Systems $\&$ Control Letters 14, pp. 103-112 (1990).

[2] K. Gilover, "All optimal Hankel-norm approximations of multivariable systems and their $L^{\infty}$ error bounds," Internat. $J$. Control 39, pp. 1115-1193 (1984).

[3] B. A. Francis, $A$ Course in $H^{\infty}$ Control Theory, Lecture Notes in Control and Information Sciences 88, Springer-Verlag, Berlin, 1986.

[4] V. Kučera, Discrete Linear Control: The Polynomial Equation Approach, Wiley, Chichester, 1979.

[5] T. Kailath, Linear Systems, Prentice Hall, Englewood Cliffs, N. J., 1980.

[6] F. M. Callier and C. A. Desoer, Multivariable Feedback Systems, Springer, Berlin, 1982.

[7] H. Kiwakernaak, "MATLAB macros for polynomial $\mathcal{H}_{\infty}$ control system optimization," Memorandum No. 881, Department of Applied Mathematics, University of Twente, September, 1990.

[8] F. M. Callier, "On polynomial matrix spectral factorization by symmetric extraction," IEEE Trans. Aut. Control 30, pp. 453-464 (1985). 